\title{
Insectos asociados al cacao en Catacamas, Honduras, 2016
}

\author{
Insects associated with cocoa in Catacamas, Honduras, 2016
}

Edgardo Jiménez Martínez ${ }^{1}$ Yoni Antúnez Munguía²

\section{Resumen}

El cacao (Theobroma cacao L.) es una planta tropical que pertenece a la familia malvaceae. Se realizó esta investigación con el objetivo de contribuir al conocimiento científico, a través de la identificación y diversidad de los insectos asociados al cultivo de cacao. El estudio se desarrolló de julio 2016 a marzo de 2017 en cuatro fincas de cacao en Honduras en el municipio de Catacamas del departamento Olancho, en las cuales se colocaron trampas de pitfall, galones con melaza, trampas amarillas y observación directa para la captura y muestreo de insectos. Los resultados han mostrado que la finca con mayor abundancia de insectos es El Nance, en total se encontraron 11 órdenes y las familias más abundantes fueron Formicidae, Muscidae, Scarabaeidae, Ceratopogonidae, Apidae, Vespidae, Nymphalidae, Culicidae, Nitidulidae y Acrididae. Según la estimación de la diversidad mediante el índice de Shannon Weaver, la finca La Vega resulta ser la más diversa con un índice de 1,06 y según el índice de Simpson la finca más dominante es Rosales.

Palabras clave: distribución temporal; abundancia; riqueza; plagas.

\section{Abstract}

Cacao (Theobroma cacao L.) is a tropical plant that belongs to the Malvaceae family. This research was carried out with the aim of contributing to scientific knowledge, through the identification and diversity of insects associated with cocoa cultivation. The study was developed from July 2016 to March 2017 in four cacao farms in Honduras in the Catacamas municipality of the Olancho department, in which pitfall traps, gallons with molasses, yellow traps and direct observation for capture and sampling were placed. of insects. The results have shown that the farm with the highest abundance of insects is El Nance, a total of 11 orders were found and the most abundant families were Formicidae, Muscidae, Scarabaeidae, Ceratopogonidae, Apidae, Vespidae, Nymphalidae, Culicidae, Nitidulidae and Acrididae. According to the diversity estimate using the Shannon Weaver index, the La Vega farm turns out to be the most diverse

\footnotetext{
PhD en Entomología, Docente Investigador, Director de Investigación, Extensión y Posgrado, Universidad Nacional Agraria, Nicaragua. Correo: edgardo.jimenez@ci.una.edu.ni ORCID: https://orcid.org/oooo-ooo3-1086-7380

2 MSc en Sanidad Vegetal. Docente Universidad Nacional Agraria
}

Recibido: 08/01/2021 - Aprobado: 05/02/2021

Jiménez Martínez, E., \& Antúnez Munguía, Y. (2021). Insectos asociados al cacao en Catacamas, Honduras, 2016. Ciencia E Interculturalidad, 28(01), 166 - 181. https://doi.org/10.5377/rci.v28i01.11467 
with an index of 1.06 and according to the Simpson index the most dominant farm is Rosales.

Keywords: Temporal Distribution; Abundance; Wealth;Pests

\section{Introducción}

El cultivo del cacao (Theobroma cacao L.) tuvo su origen en América, pero no se puede indicar con precisión el lugar específico ni su distribución, el cacao es uno de los cultivos alimenticios que desde el punto de vista tecnológico e industrial ha tenido un avance más lento. Quizás una de las razones se debe a su carácter altamente minifundista y las características de incompatibilidad genética que lo caracterizan (Batista, 2009).

Honduras es considerado como uno de los pocos países privilegiados del mundo en contar con cultivares superiores tipo trinitarios (Fundación Hondureña de Investigación Agrícola, 2004), así como con materiales criollos nativos que conservan su pureza genética, por el hecho de estar aislados y por la interacción genotipo-ambiente que les confiere un sabor y aroma especial, que es apetecido por las firmas chocolateras más prestigiosas del mundo que elaboran chocolate gourmet (Dubón y Sánchez, 2011).

En el cacao, debido a que el polen es pegajoso y tiene alto contenido de humedad, es difícil que el viento pueda transportarlo (Cabezas et al., 2013). Es muy difícil que el polen llegue transportado por el viento porque las anteras están tapadas por los pétalos y se encuentran más abajo que el estilo o el estigma de la flor, por lo que los polinizadores se constituyen agentes fundamentales en estos sistemas, principalmente los insectos. Estudios en varias regiones productoras de cacao alrededor del mundo han demostrado que los polinizadores del cacao pertenecen principalmente a la orden díptera, siendo la familia Ceratopogonidae (géneros Forcipomyia, Dasyhelea y Atrichopogon) de mayor importancia en regiones como Malasia, Ghana y Costa Rica (Ríos, 2015).

En Honduras no existe información relacionada con la diversidad de insectos del cacao; sin embargo, algunos trabajos realizados solamente incluyen aspectos fitosanitarios, plagas y polinizadores. Con la realización de esta investigación se pretende obtener una base de conocimiento científico sobre los principales insectos asociados al cultivo de cacao en Honduras, información que podrá ser utilizada por los productores nacionales e investigadores que deseen hacer uso de esta herramienta. 


\section{Materiales y métodos}

\section{Ubicación y fecha de estudio}

El estudio se realizó de julio de 2016 a marzo del 2017 en cuatro fincas de cacao en producción. Las fincas se ubican en el municipio de Catacamas del departamento de Olancho,Honduras; dos de ellas se ubican en el campus de la Universidad Nacional de Agricultura y las otras en la aldea de Río Tinto a $20 \mathrm{~km}$ al sureste de la ciudad de Catacamas. La temperatura media es de $26.6^{\circ} \mathrm{C}$ con precipitación promedio anual de $1500 \mathrm{~mm}$ anuales.

Cuadro no. 1: Datos de localización de las fincas de cacao evaluadas en el municipio de Catacamas, Olancho

\begin{tabular}{lllllll}
\hline \multicolumn{1}{c}{ Finca } & \multicolumn{1}{c}{ Productor } & Comunidad & Área (ha-1) & Latitud & Longitud & msnm \\
\hline El Nance & UNA & El Espino & 1.2 & 1638647 & 624589 & 354 \\
Guanacaste & UNA & El Espino & 0.20 & 1639016 & 624418 & 362 \\
La Vega & Pedro & Rio Tinto & 1.5 & 1650277 & 641841 & 376 \\
& Alemán & & & & & \\
Rosales & Orlando & Rio Tinto & 2.2 & 1651699 & 637758 & 412 \\
& Rosales & & & & & \\
\hline
\end{tabular}

\section{Metodología para el muestreo de insectos}

En cada finca se seleccionaron tres sitios de muestreo separados a una distancia aproximada de $20 \mathrm{~m}$ ubicando trampas para la captura de insectos, se colocaron tres trampas pitfall traps, tres trampas del tipo galones de plástico y tres trampas amarillas pegajosas, el conteo de insectos se realizó en las partes aéreas de la planta (tallo, hoja, flores y frutos) semanalmente de la siguiente manera:

El primer método consistió en la captura de insectos rastreros y algunos insectos voladores con trampas pitfall traps, estas trampas son recipientes o panas plásticas de $30 \mathrm{~cm}$ de diámetro y $15 \mathrm{~cm}$ de profundidad, puestas a ras del suelo con capacidad de cuatro litros de agua, se le agregó cuatro gramos de detergente y cuatro gramos de bórax (ácido bórico) para preservar la solución.

En el segundo método para insectos voladores, se utilizó trampas de galones plásticos cortados por uno de sus costados con capacidad de cuatro litros de agua y se le adicionaron $10 \mathrm{ml}$ de melaza, que cumplió la función de atrayente. Estos recipientes se colocaron horizontalmente sujetados en una de las ramas del árbol a una altura de metro y medio sobre el suelo. 
El tercer método fue la ubicación de trampas amarillas pegajosas con aceite vegetal en las ramas cercas a las flores de la planta, para la captura de insectos polinizadores y otros insectos de menor tamaño. Se realizó el conteo de insectos visualmente en dos ramas de dos árboles ubicados frente a la planta que contiene las trampas de galones plásticos. La inspección en las trampas se realizó semanalmente, dándole mantenimiento, limpiándolas y cambiando la solución una vez por semana.

\section{Muestras e identificación de insectos en laboratorio}

Los insectos colectados fueron ubicados en viales entomológicos con alcohol al $75 \%$, se etiquetaron y se identificaron para clasificarlos en el laboratorio de entomología de la Universidad Nacional de Agricultura de Honduras y la Universidad Nacional Agraria de Nicaragua, utilizando claves dicotómicas y comparación con otros insectos clasificados, según consultas bibliográficas en el texto básico: Entomología (Jiménez Martínez, 2009), Guía para el estudio de: Ordenes y familias de insectos de Centroamérica (Andrews y Caballero, 1989), Insectos de Nicaragua: Catalogo de los Insectos y Artrópodos Terrestres de Nicaragua (Maes, 1998) y Entomología Sistemática (Sáenz y De la Llana, 1990).

\section{Variables evaluadas}

\section{Abundancia total de insectos por finca}

Se tomaron datos desde el 18 de julio de 2016, una vez por semana hasta el 31 de marzo de 2017, realizando un conteo de todos los insectos encontrados por finca en cada una de las trampas.

\section{Riqueza de familias de insectos por finca}

Se registró el número total de especies de cada familia durante el tiempo de muestreo en las cuatro fincas evaluadas.

\section{Índice de diversidad de Shannon Weaver}

Esta variable se calcula mediante el índice de diversidad de Shannon Weaver (1949), utilizando la siguiente fórmula:

$$
H^{\prime}=-\sum_{i=1}^{S} p_{i} \log _{2} p_{i}
$$

Dónde: 
- $H=$ Número de especies (riqueza de especies).

- $P i=$ Abundancia proporcional de la iésima especie $(n i / N)$.

- $n i=$ Número de individuos de la especie $i$.

- $N=$ Número de todos los individuos de todas las especies.

- Índice de diversidad de Simpson

Es el número de individuos de especies capturadas por hábitat utilizando el índice de Simpson con criterios de heterogeneidad. Para el cálculo del índice de diversidad se utilizó la siguiente formula:

Dónde:

- $p i=n i / N$

- $n i=$ representa la abundancia de la especie $i$.

- $N=$ el número total de individuos.

Simpson mide la dominancia, es decir, da valores de dominancia dentro de una comunidad, por tal motivo se le resto 1 para estimar la diversidad. Es decir: (Simpson, 1949).

\section{Análisis de los datos}

Colectados los datos, estos fueron arreglados por variables de familias de insectos por finca en una tabla de datos en Excel, donde se ordenan todas las variables para su análisis, graficado y comparado entre fincas. Así mismo se realizan los diferentes cálculos de diversidad de insectos usando el índice de Shannon-Weaner y el índice de Simpson.

\section{Resultados y discusión}

Los insectos asociados al cultivo de cacao en el departamento de Olancho, municipio de Catacamas, utilizando diferentes métodos de trampeo en varias fincas de cacao, están distribuidos en 11 órdenes, 52 familias y 87 géneros, con un total de 13,393 insectos (Cuadro 2). 
Cuadro no. 2: Principales órdenes, familias, géneros, especies y habito alimenticio de insectos encontrados en el cultivo de cacao

\begin{tabular}{|c|c|c|c|c|c|}
\hline Orden & Familia & Género & Especie & Nombre común & Hábito alimenticio \\
\hline \multirow{12}{*}{ Díptera } & Asilidae & Efferia & $s p$ & Mosca ladrona & Depredador \\
\hline & Calliphoridae & Cochliomyia & $\begin{array}{c}\text { hominivorax } \\
\text { C. }\end{array}$ & Moscas & Saprófago \\
\hline & Ceratopogonidae & Forcipomyia & $s p$ & $\begin{array}{c}\text { Mosquita del } \\
\text { cacao }\end{array}$ & Nectarívoro \\
\hline & \multirow{2}{*}{ Culicidae } & Aedes & aegyptiL. & Zancudos & Hematófago \\
\hline & & Anopheles & sp. & Zancudos & Hematófago \\
\hline & Drosophilidae & Drosophila & $s p$ & Drosofilidos & Saprófago \\
\hline & Muscidae & Musca & domestica L. & Mosca & Saprófago \\
\hline & Otitidae & Euxesta & $s p$ & Alas pintadas & $\begin{array}{l}\text { Nectarívoro } \\
\text { (hematófago) }\end{array}$ \\
\hline & Simuliidae & Simulium & sp. & Jejenes & Hematófago \\
\hline & Tabanidae & Tabanus & sp. & Tábano & Nectarívoro \\
\hline & Tachinidae & Lespecia & sp. & Mosca & Fitófago \\
\hline & Tephritidae & Anastrepha & sp. & $\begin{array}{c}\text { Mosca de la } \\
\text { fruta }\end{array}$ & Fitófago \\
\hline \multirow{4}{*}{ Orthoptera } & \multirow[b]{2}{*}{ Acrididae } & Taeniopoda & sp. & Saltamontes & Fitófago \\
\hline & & Dichromorpha & viridis S. & Saltamontes & Fitófago \\
\hline & Gryllidae & Gryllus & $s p$. & Grillo & Fitófago \\
\hline & Tettigoniidae & Neoconocephalus & sp. & Esperanza & Fitófago \\
\hline
\end{tabular}




\begin{tabular}{|c|c|c|c|c|c|}
\hline Orden & Familia & Género & Especie & Nombre común & Hábito alimenticio \\
\hline \multirow{25}{*}{ Coleoptera } & Cerambycidae & No identificado & sp. & Antenudo & Xilófago \\
\hline & \multirow{4}{*}{ Chrysomelidae } & Omophoita & $s p$. & Tortuguilla & Fitófago \\
\hline & & Altica & sp. & Tortuguilla & Fitófago \\
\hline & & Acalymma & $s p$. & Tortuguilla & Fitófago \\
\hline & & Diabrotica & $s p$. & Tortuguilla & Fitófago \\
\hline & \multirow{2}{*}{ Coccinellidae } & Cycloneda & sanguinea $L$. & Mariquita & Depredador \\
\hline & & Chilocorus & $s p$. & Mariquita & Depredador \\
\hline & Dermestidae & Dermestes & $s p$. & Gorgojo & Saprofitos \\
\hline & \multirow{4}{*}{ Curculionidae } & Cosmopolites & sordidus G. & $\begin{array}{l}\text { Picudo del } \\
\text { plátano }\end{array}$ & Fitófago \\
\hline & & Rhynchophorus & palmarum L. & $\begin{array}{c}\text { Picudo de la } \\
\text { palma }\end{array}$ & Fitófago \\
\hline & & Hypothenemus & hampeif. & Broca & Fitófago \\
\hline & & xyleborus & $s p$ & Barrenador & Fitófago \\
\hline & Elateridae & Agriotes & $s p$. & Escarabajo clik & Fitófago \\
\hline & \multirow{2}{*}{ Lampyridae } & Aspisoma & sp. & Luciérnaga & Depredador \\
\hline & & Photinus & sp. & Luciérnaga & Depredador \\
\hline & \multirow{4}{*}{ Nitidulidae } & Colopterus & $s p$. & Nitidulidos & Saprofitos \\
\hline & & Lobiopa & $s p$. & Nitidulidos & Saprofitos \\
\hline & & Nitidula & sp. & Nitidulidos & Saprofitos \\
\hline & & Cryptarcha & $s p$. & Gorgojo & Saprofitos \\
\hline & Passalidae & Passalus & sp. & Pasalidos & Fitófago \\
\hline & \multirow{4}{*}{ Scarabaeidae } & Phyllophaga & $s p$. & Escarabajo & Fitófago \\
\hline & & Copris & sp. & Escarabajo & Coprófago \\
\hline & & Euphoria & sp. & Escarabajo & Fitófago \\
\hline & & Dichotomius & sp. & Escarabajo & Coprófago \\
\hline & Tenebrionidae & Lobometopon & sp. & Escarabajo & Fitófago \\
\hline
\end{tabular}




\begin{tabular}{|c|c|c|c|c|c|}
\hline Orden & Familia & Género & Especie & Nombre común & Hábito alimenticio \\
\hline \multirow{13}{*}{ Hymenóptera } & \multirow{4}{*}{ Apidae } & Apis & melliphera L. & Abeja & Nectarívoro \\
\hline & & Mellipona & $s p$ & Morroco & Nectarívoro \\
\hline & & Xylocopa & sp. & Abejorro & Nectarívoro \\
\hline & & Trygona & $s p$ & Jimerito & Nectarívoro \\
\hline & \multirow{3}{*}{ Formicidae } & Atta & $s p$ & Zompopo & Fitófago \\
\hline & & Crematogaster & $s p$ & Hormiga & Depredador \\
\hline & & Lasius & niger L. & Hormiga & Depredador \\
\hline & Sphecidae & Sceliphron & $s p$ & Avispa & Depredador \\
\hline & Tenthredinidae & Macrophya & $s p$ & Avispita & Fitófago \\
\hline & Ichneumonidae & Eiphosoma & $s p$ & Avispita & Parasitoide \\
\hline & \multirow{3}{*}{ Vespidae } & Polybia & $s p$ & Avispa & Depredador \\
\hline & & Polistes & $s p$ & Avispa & Depredador \\
\hline & & Synoeca & sp. & Avispa & Depredador \\
\hline \multirow{9}{*}{ Lepidóptera } & Geometridae & No identificado & $s p$ & Polilla & Fitófago \\
\hline & Lycaenidae & Strymon & $s p$ & Licaenidos & Fitófago \\
\hline & \multirow{3}{*}{ Nymphalidae } & Anarthia & $s p$. & Mariposa & Fitófago \\
\hline & & Dione & $s p$ & Mariposa & Fitófago \\
\hline & & Heliconius & $s p$ & Mariposa & Fitófago \\
\hline & Noctuidae & Spodoptera & $s p$ & Polilla & Fitófago \\
\hline & \multirow{3}{*}{ Pieridae } & Pieris & $s p$ & Mariposa & Fitófago \\
\hline & & Phoebis & $s p$ & Mariposa & Fitófago \\
\hline & & Leptophobia & aripa & $\begin{array}{c}\text { Mariposa blan- } \\
\text { ca }\end{array}$ & Fitófago \\
\hline \multirow{3}{*}{ Blattodea } & \multirow{3}{*}{ Blattidae } & Panchlora & $s p$ & Cucaracha & Omnívoros \\
\hline & & Blaberus & $s p$ & Cucaracha & Omnívoros \\
\hline & & Periplaneta & americana L. & Cucaracha & Omnívoros \\
\hline
\end{tabular}




\begin{tabular}{|c|c|c|c|c|c|}
\hline Orden & Familia & Género & Especie & Nombre común & Hábito alimenticio \\
\hline \multirow{15}{*}{ Hemiptera } & \multirow{3}{*}{ Pentatomidae } & Nezara & viridula L. & Chinche & Fitófago \\
\hline & & Podisus & sp. & Chinche & Fitófago \\
\hline & & Edessa & sp. & Chinche & Fitófago \\
\hline & Gelastocoridae & Gelastocoris & sp. & Chinche sapo & Depredador \\
\hline & Cimicidae & Cimex & sp. & $\begin{array}{c}\text { Chinche de } \\
\text { cama }\end{array}$ & Hematofago \\
\hline & \multirow[t]{2}{*}{ Reduviidae } & Triatoma & dimidiata Lat. & $\begin{array}{c}\text { Chinche pi- } \\
\text { cuda }\end{array}$ & Hematófago \\
\hline & & Zelus & sp. & Chinche & Depredador \\
\hline & Membracidae & Membracis & sp. & Periquitos & Fitófago \\
\hline & \multirow[b]{2}{*}{ Coreidae } & Pachylis & sp. & Chinche & Fitófago \\
\hline & & Leptoglossus & sp. & $\begin{array}{c}\text { Chinche pata } \\
\text { de hoja }\end{array}$ & Fitófago \\
\hline & Cicadellidae & Empoasca & sp. & Lorito verde & Fitófago \\
\hline & Aphididae & Aphis & sp. & Pulgones & Fitófago \\
\hline & \multirow{2}{*}{ Cercopidae } & Prosapia & sp. & Salivazo & Fitófago \\
\hline & & Aeneolamia & sp. & Salivazo & Fitófago \\
\hline & Cicadidae & Diceroprocta & sp. & Chicharra & Fitófago \\
\hline \multirow[b]{2}{*}{ Odonata } & Libelullidae & Libellula & sp. & Libélula & Depredador \\
\hline & Coenagrionidae & Argia & sp. & $\begin{array}{c}\text { Caballitos del } \\
\text { diablo }\end{array}$ & Depredador \\
\hline Thysanoptera & Thripidae & Thrips & sp. & Trips & Fitófago \\
\hline Neuróptera & Chrysopidae & Chrysopa & sp. & Crisopa & Depredador \\
\hline Isóptera & Termitidae & Nasutitermes & sp. & Termitas & Xilófagos \\
\hline
\end{tabular}

\section{Abundancia total de insectos}

La abundancia total de insectos encontrados fue de 13,393. Resultando la finca El Nance con el mayor número de insectos con un total de 3 617, finca La Vega 3394 , finca Los Guanacastes con 3341 y la finca Rosales fue la que presentó el menor número con 3224 (Figura 1). La finca El Nance fue donde se presentó la mayor cantidad de insectos, seguido por finca La Vega, esto se relaciona a la abundancia de vegetación (malezas de hoja ancha) que predominó durante el periodo de evaluación. 


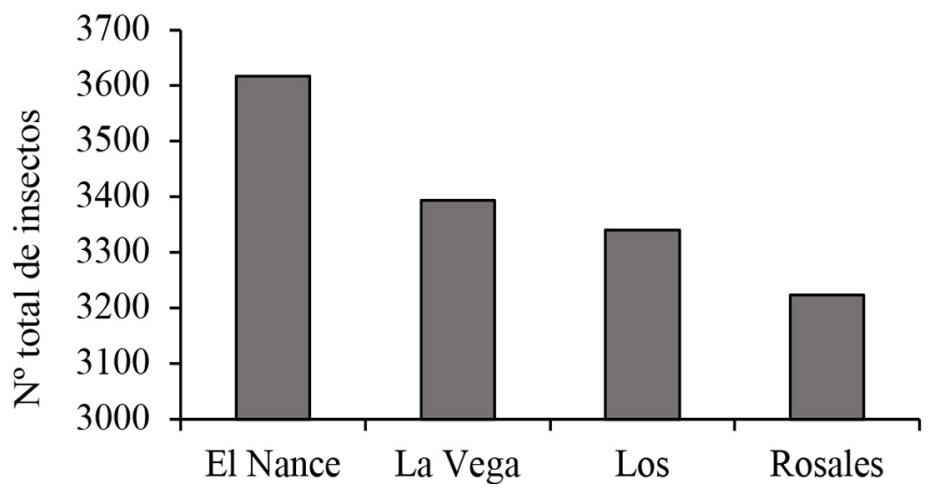

Figura no. 1. Abundancia total de insectos encontrados en las cuatro fincas de cacao.

La mayor cantidad de insectos encontrados en todos los muestreos realizados en las cuatro fincas pertenecen a los órdenes Hymenóptera, Díptera, Coleóptera, Lepidóptera Hemíptera y Orthóptera. Sarcus (2016), en un inventario de fauna entomológica asociada al cultivo de cacao encontró el $96.5 \%$ de los insectos pertenecientes a los órdenes Coleóptera, Hymenóptera y Lepidóptera.

En un estudio reciente para determinar la diversidad de polinizadores desarrollado en África, se realizaron importantes colecciones de insectos en época de floración. En este contexto, se catalogaron 2,721 insectos pertenecientes a 7 órdenes y 36 especies, pero de éstas únicamente aquellas aladas fueron las que se reportaron asociadas a las flores del cacao (Adjaloo y Oduro, 2013).

La mayor cantidad de insectos en La Vega pertenecen a las familias Muscidae, Apidae, Culicidae y Acrididae mientras que en Rosales corresponden a las familias Formicidae y Ceratopogonidae, en Los Guanacastes la mayor cantidad pertenecen a Scarabaeidae y Nitidulidae y en El Nance la mayor abundancia se encuentra distribuida en las familias Vespidae y Nymphalidae. En este trabajo el mayor número de insectos son de la familia Formicidae, insectos que en el cacao se alimentan del follaje (Atta sp.) y son depredadores de áfidos (Crematogaster y Lasius sp.), en su mayoría estos insectos se capturaron en trampas de pitfall traps ya que son de habito terrestre y caminan sobre la superficie del suelo.

\section{Riqueza de familias de insectos por finca}

En la finca La Vega se encontró el mayor número de familias de insectos con un total de 52 y 51 para Rosales, en El Nance y Los Guanacastes se encontró un menor número con un total de 45 y 41 respectivamente (Figura 2). La finca La Vega se caracterizó por presentar mayor cobertura vegetal (malezas de hoja ancha) durante 
el periodo de evaluación y se asume este factor como la posible causa de una mayor presencia de insectos respecto a las demás fincas.

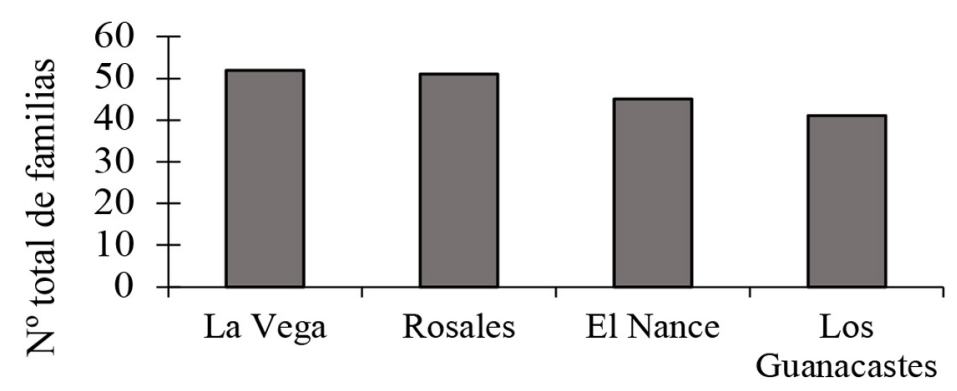

Fincas

Figura no. 2. Riqueza total de familias de insectos encontrados en cuatro fincas de cacao.

Rivero (2006) realizó un estudio de diversidad de insectos en la región JibacoaHanabanilla, Cuba, donde se encontraron 61 familias. En el cultivo de marañón en Nicaragua, Rugama y López (2011) reportaron una riqueza de 35 familias y Gómez (2011), una riqueza total de 11 familias. Así mismo, Lacayo y Mayorga (2014) encontraron una riqueza de 15 familias en el cultivo de marango.

Montano y Bustamante (2017) muestran una riqueza de 32 familias en el cultivo de maracuyá y Mairena (2015) en su investigación del cultivo de piña, la riqueza de insectos encontradas en el período de marzo a septiembre del 2014 fue de 51 familias, datos similares a los presentados en esta investigación en el cultivo de cacao.

\section{Índice de diversidad de Shannon Weaver}

El índice de Shannon o índice de Shannon-Weaver se representa normalmente como H'y se expresa con un número positivo, que en la mayoría de los ecosistemas naturales varía entre 0,5 y 5 aunque su valor normal está entre 2 y 3 . Los ecosistemas con mayores valores son los bosques tropicales y arrecifes de coral, y los menores las zonas desérticas (Shannon, 1949).

Los promedios de diversidad según Shannon en este estudio resultan ser bajos, con valores de 1,05 para Rosales y Los Guanacastes y 1,06 para La Vega y El Nance, considerados como un ecosistema de baja biodiversidad, ya que Shannon (1949) considera que valores inferiores a 2 representa zonas de baja biodiversidad y superiores a 3 zonas de alta biodiversidad. 
Cuadro no. 3: Índice de diversidad de Shannon-Weaver de las principales familias de insectos asociados al cultivo de cacao.

\begin{tabular}{|c|c|c|c|c|}
\hline \multirow{2}{*}{ FAMILIA } & \multicolumn{4}{|c|}{ Índice de diversidad de Shannon-Weaver } \\
\hline & Rosales & La Vega & Los Guanacastes & El Nance \\
\hline Formicidae & 1,43 & 1,37 & 1,41 & 1,39 \\
\hline Muscidae & 1,31 & 1,34 & 1,30 & 1,28 \\
\hline Scarabaeidae & 1,24 & 1,22 & 1,24 & 1,23 \\
\hline Ceratopogonidae & 1,21 & 1,22 & 1,18 & 1,18 \\
\hline Apidae & 1,16 & 1,18 & 1,17 & 1,17 \\
\hline Vespidae & 1,13 & 1,18 & 1,16 & 1,15 \\
\hline Noctuidae & 1,11 & 1,15 & 1,16 & 1,15 \\
\hline Culicidae & 1,11 & 1,15 & 1,14 & 1,13 \\
\hline Aphididae & 1,09 & 1,13 & 1,14 & 1,13 \\
\hline Lycaenidae & 1,09 & 1,11 & 1,12 & 1,12 \\
\hline Reduviidae & 1,09 & 1,08 & 1,12 & 1,12 \\
\hline Curculionidae & 1,08 & 1,07 & 1,11 & 1,11 \\
\hline Nymphalidae & 1,08 & 1,07 & 1,10 & 1,09 \\
\hline Calliphoridae & 1,07 & 1,06 & 1,08 & 1,09 \\
\hline Acrididae & 1,07 & 1,06 & 1,05 & 1,08 \\
\hline Nitidulidae & 1,05 & 1,06 & 1,05 & 1,06 \\
\hline Lampyridae & 1,05 & 1,05 & 1,04 & 1,06 \\
\hline Gelastocoridae & 1,04 & 1,05 & 1,04 & 1,06 \\
\hline Otitidae & 1,04 & 1,04 & 1,04 & 1,04 \\
\hline Blattidae & 1,04 & 1,04 & 1,04 & 1,04 \\
\hline Coenagrionidae & 1,03 & 1,04 & 1,04 & 1,04 \\
\hline Chrysopidae & 1,03 & 1,03 & 1,04 & 1,03 \\
\hline Pieridae & 1,03 & 1,03 & 1,03 & 1,03 \\
\hline Drosophilidae & 1,03 & 1,03 & 1,03 & 1,03 \\
\hline Gryllidae & 1,03 & 1,03 & 1,03 & 1,03 \\
\hline Thripidae & 1,03 & 1,03 & 1,03 & 1,03 \\
\hline Elateridae & 1,02 & 1,03 & 1,02 & 1,03 \\
\hline Membracidae & 1,02 & 1,03 & 1,02 & 1,03 \\
\hline Tettigoniidae & 1,02 & 1,03 & 1,02 & 1,02 \\
\hline Chrysomelidae & 1,02 & 1,02 & 1,02 & 1,02 \\
\hline Coreidae & 1,02 & 1,02 & 1,01 & 1,02 \\
\hline Passalidae & 1,01 & 1,02 & 1,01 & 1,02 \\
\hline
\end{tabular}




\begin{tabular}{|l|l|l|l|l|}
\hline \multirow{2}{*}{ FAmILIA } & \multicolumn{4}{|c|}{ Índice de diversidad de Shannon-Weaver } \\
\cline { 2 - 5 } & Rosales & La Vega & Los Guanacastes & El Nance \\
\hline Pentatomidae & 1,01 & 1,02 & 1,01 & 1,02 \\
\hline Tachinidae & 1,01 & 1,02 & 1,01 & 1,02 \\
\hline Coccinellidae & 1,01 & 1,02 & 1,01 & 1,02 \\
\hline Tenebrionidae & 1,01 & 1,02 & 1,01 & 1,02 \\
\hline Sphecidae & 1,01 & 1,02 & 1,01 & 1,01 \\
\hline Dermestidae & 1,01 & 1,01 & 1,01 & 1,01 \\
\hline Cerambycidae & 1,01 & 1,01 & 1,01 & 1,01 \\
\hline Geometridae & 1,01 & 1,01 & 1,00 & 1,01 \\
\hline Cimicidae & 1,01 & 1,01 & 1,00 & 1,01 \\
\hline Cercopidae & 1,01 & 1,01 & 1,00 & 1,00 \\
\hline Tabanidae & 1,01 & 1,01 & 1,00 & 1,00 \\
\hline Cicadellidae & 1,01 & 1,01 & 1,00 & 1,00 \\
\hline Libelullidae & 1,01 & 1,01 & 1,00 & 1,00 \\
\hline Ichneumonidae & 1,01 & 1,01 & 1,00 & 1,00 \\
\hline Asilidae & 1,01 & 1,01 & 1,00 & 1,00 \\
\hline Simuliidae & 1,01 & 1,00 & 1,00 & 1,00 \\
\hline Tenthredinidae & 1,00 & 1,00 & 1,00 & 1,00 \\
\hline Tephritidae & 1,00 & 1,00 & 1,00 & 1,00 \\
\hline Cicadidae & 1,00 & 1,00 & 1,00 & 1,00 \\
\hline Termitidae & 1,00 & 1,00 & 1,00 & 1,00 \\
\hline & 1,0572 & 1,0610 & $\mathbf{1 , 0 5 8 3}$ & $\mathbf{1 , 0 6 0 7}$ \\
\hline
\end{tabular}

El índice refleja la heterogeneidad de una comunidad sobre la base de dos factores: el número de especies presentes y su abundancia relativa. Conceptualmente es una medida del grado de incertidumbre asociada a la selección aleatoria de un individuo en la comunidad (Pla, 2006).

\section{Índice de diversidad de Simpson}

El índice de Simpson mide la probabilidad de que dos individuos de la población extraídos al azar sean de la misma especie $\left(\sum p i^{2}\right)$ la probabilidad promedio de que ocurra será igual a la suma de las probabilidades individuales de cada especie según al hábitat perteneciente, valores altos indican dominancia de alguna especie. Los valores varían de o a 1 indicando que los valores próximos a 1 representan mayor diversidad. Este índice ofrece información intermedia entre el índice de Shannon y el de uniformidad, ya que aumenta con el número de especies y refleja a su vez el reparto de las especies en proporciones (Simpson, 1949). 
Se presentó mayor dominancia de especies por familias de insectos en la finca Rosales (o,012) (Cuadro 4). Por su parte el menor índice de diversidad de Simpson lo tiene la finca Rosales y los mayores valores de diversidad lo presentan las fincas La Vega, El Nance y Los Guanacastes. Este índice de diversidad (1-D), es inversamente proporcional a la Dominancia (D), por lo que las fincas La Vega, El Nance y Los Guanacastes son menos diversas en número de especies por familias, pero más dominantes.

Cuadro no. 4: Índice de diversidad de Simpson de las principales familias de insectos asociados al cultivo de cacao

\begin{tabular}{|l|l|l|}
\hline \multicolumn{1}{|c|}{ Finca } & \multicolumn{1}{c|}{ Dominancia } & \multicolumn{1}{c|}{ Índice de diversidad de Simpson } \\
\hline La Vega & 0,087 & 0,913 \\
\hline El Nance & 0,088 & 0,912 \\
\hline Los Guanacastes & 0,099 & 0,900 \\
\hline Rosales & 0,012 & 0,878 \\
\hline
\end{tabular}

\section{Conclusiones}

Se encontró un total de 11 órdenes de insectos, 52 familias y 87 géneros en las cuatro fincas estudiadas, las familias más dominantes fueron Formicidae, Muscidae, Scarabaeidae, Ceratopogonidae y Apidae.

La mayor abundancia de insectos asociados al cultivo de cacao se presentó en la finca El Nance, sin embargo, la mayor riqueza de familias y el mayor valor de índice de diversidad de Simpson fue encontrado en finca La Vega.

\section{Lista de referencias}

Adjaloo, M., y Oduro, W. (2013). Insect assemblage and the pollination System of cocoa (Theobroma cacao L). Journal of applied Bioscience.

Andrews Keith. L., y Caballero, R. (1989). Guía para el estudio de órdenes y familias de insectos de Centroamérica. Francisco Morazán, Honduras: Escuela Agrícola Panamericana El Zamorano.

Batista, L. (2009). Guía técnica el cultivo de cacao. http://www.rediaf.net.do/publicaciones/guias/download/cacao.pdf

Cabezas, S. R., Mavisoy, H., Somarriba, E., Cerda, E., Pérez, G., y Ballesteros, W. (2013). Evaluación de la abundancia de polinizadores de cacao (Theobroma cacao L.) en la hojarasca de siete especies de sombra en cacaotales de Talamanca, Costa 


\section{AGROPECUARIA}

Rica. https://soclaperu.files.wordpress.com/2013/10/259-evaluacic3b3n-de-laabundancia-de-polinizadores-de-cacao-theobroma-cacao-l-en-la-hojarasca-desiete-especies-de-sombra-en-cacaotales-de-talamanca-costa.pdf

Dubón, A., y Sánchez, J. (2011). Manual de producción de cacao. La lima, Cortes, Honduras: FHIA.

Fundación Hondureña de Investigación Agrícola (2004). Cultivo de cacao bajo sombra de maderables y frutales. http://www.fhia.org.hn/dowloads/cacao_pdfs/cultivo_de_cacao_bajo_sombra_de_maderables_o_frutales.pdf

Gómez Martínez, J. (2011). Entomofauna y patógenos asociados al cultivo de marañón (Anacardium occidentale L.), en León, Nicaragua, entre los meses de julio 2009 a marzo 2010. (Tesis de maestría). Repositorio institucional Universidad Nacional Agraria. http://repositorio.una.edu.ni/2167/1/tnh1og633p.pdf

Jiménez Martínez, E. S. (2009). Texto Básico: Entomología. Managua Nicaragua: UNA.

Lacayo Rodríguez, R. T., y Mayorga Mendoza, J. R. (2014). Abundancia, riqueza y diversidad insectil asociada al cultivo de moringa (Moringa oleifera L.) en Managua, Nicaragua, Entre los meses de mayo a diciembre del 2013. (Tesis de grado). Repositorio Institucional Universidad Nacional Agraria http://repositorio. una.edu.ni/3156/1/tnh1ol129a.pdf

Maes, J.M. (1998). Insectos de Nicaragua: catálogo de los insectos y artrópodos terrestres de Nicaragua. León, Nicaragua.

Mairena Vásquez, C. L. (2015). Identificación y fluctuación poblacional de insectos asociados al cultivo de la piña (Ananas comusus L. Merril.), en Ticuantepe, Nicaragua, Entre los meses de marzo a septiembre 2014. (Tesis de maestría). Repositorio Institucional Universidad Nacional Agraria. http://repositorio.una.edu. ni/3363/1/tnh1om228.pdf

Montano Núñez, R. G., y Bustamante Maradiaga, E. J. (2017). Entomofauna, diversidad y fluctuación poblacional de insectos plagas y benéficos asociados al cultivo del maracuyá (Passiflora edulis Sims), en Sébaco, Matagalpa, 2016. (Tesis de grado). Repositorio Institucional Universidad Nacional Agraria http://repositorio. una.edu.ni/3456/1/tnh1om765.pdf

Pla, L. (2006). Biodiversidad: Inferencia basada en el índice de Shannon y la riqueza. SciELO. 31(8), 583-590. 
Ríos, D. F. (2015). Descripción de la diversidad entomológica asociada a la flor de Theobroma cacao (Tesis de grado). Pontífice Universidad Católica de Ecuador, Quito, Ecuador.

Rivero Aragón, A. (2006). Estudios de diversidad de insectos en la región JibacoaHanabanilla. Macizo Guamuhaya. http://cagricola.uclv.edu.cu/descargas/pdf/ V33-Numero_2/cago82061483.pdf

Rugama Lovo, I. M., y López Vílchez, M. E. (2011). Identificación y descripción de los principales insectos rastreros asociados al cultivo de marañón (Anacardium occidentale L.), orgánico y convencional en León, Nicaragua, durante los meses de agosto 2009 a marzo 2010. (Tesis de grado) http://repositorio.una.edu.ni/2142/1/ tnh1org28i.pdf

Sáenz, M., y De La Llana, A. (1990). Entomología sistemática. Managua, Nicaragua: UNA.

Sarcus, C.E. (2016). Inventario de fauna entomológica asociado al cultivo de cacao (Theobroma caao L.) en una plantación con sistema agroforestal en el Cantón, Baba, recinto La Carmela, provincia de Los Ríos (Tesis de grado). Universidad Técnica de Babahoyo. Los Ríos, Ecuador.

Shannon, C. E y Weaver, W. (1949). The mathematical theory of communication. Illinois, Estados Unidos: University of Illinois Press.

Simpson, E.H. (1949). Measurement of diversity. Nature 163, 688. 